\title{
Live My Work: rural nurses and their multiple perspectives of self
}

\begin{abstract}
Aim: This paper is a report of a study that explored rural nurses' experiences of mentoring.

Background: Mentoring has recently been proposed by governments, advocates and academics as a solution to the problem of workforce for Australian rural nurses. Action in the form of mentor development workshops has changed the way that some rural nurses now construct supportive relationships as mentoring.

Method: A grounded theory design was used with nine rural nurses. Eleven semi-structured interviews were conducted in various states of Australia during 2004 and 2005. Situational analysis mapping techniques and frame analysis were used in combination with concurrent data generation and analysis and theoretical sampling.

Findings: Experienced rural nurses cultivate novices through supportive mentoring relationships. The impetus for such relationships comes from their own histories of living and working in the same community, and this was termed 'live my work'. Rural nurses use multiple perspectives of self in order to manage their interactions with others in their roles as community members, consumers of healthcare services and nurses. Personal strategies adapted to local context constitute the skills that experienced rural nurses pass on to neophyte rural nurses through mentoring, while at the same time protecting them through troubleshooting and translating local cultural norms.
\end{abstract}

\section{Conclusion:}

Living and working in the same community creates a set of complex challenges for novice rural nurses that are better faced with a mentor in place. Thus, mentoring has become an integral part of experienced rural nurses' practice to promote staff retention.

\section{Key Words}

Grounded Theory, Rural Nursing, Mentors, Support, Workforce Issues, Interviews, Frame Analysis 


\section{Summary Statements}

\section{What is already known about this topic}

- $\quad$ Living and working in the same community creates a set of complex challenges for rural nurses

- Mentoring is a strategy used to retain novice rural nurses

\section{What this paper adds}

- Rural nurses use multiple perspectives of self to manage living and working in the same community

- Mentoring novice rural nurses is framed using the lenses of culture, politics and clinical practice

- Mentoring strategies used by experienced rural nurses and the expected outcomes from these strategies include orientation to local cultural mores and an increase in the neophyte nurses' confidence to practice. 


\section{Introduction}

Rural nurses are those who practise outside of major metropolitan centres, where access to goods and services such as health care is reduced. These nurses may practise either with or without medical and/or allied healthcare professional support (Francis et al. 2002).

Rural nursing gained momentum as a specialty area of nursing in the late 1980s and early 1990s. Living and working in the same community has been framed both positively and negatively in the literature (Hegney and McCarthy 2002). For some rural nurses, losing their anonymity and often knowing their 'patient[s] and their families because of the closeness of the community often leads to stress' (LeSergent and Haney 2005, p.321). For other rural nurses, the most positive part of working in the country is being a central part of a strong social network that often involves family as well as friends (Hegney et al. 2002).

Internationally, mentoring has emerged as a support strategy for career advancement in nursing in the 1970s mainly in the United States of America (USA) (Vance and Olson 1998). Since that time, the concept of mentoring has evolved in the literature as either a formal or informal process.

\section{Background}

In Australia, Francis et al. (2002) argue that the Rural, Remote and Metropolitan (RRMA) classification is the most appropriate framework to use when classifying rural nursing because it accounts for greater variance in the characteristics of the populations studied. RRMAs index of remoteness is based on distance to service centres as well as a measure of distance from other people (Australian Institute of Health and Welfare 2004). Living in rural Australia often means that healthcare clients requiring advanced specialty services such as cardiac surgery, transplantation, paediatric and most cancer treatments are required to travel long distances, with many services being available only in major cities.

Research has been undertaken into the experiences of Australian rural nurses to identify characteristics that differentiate rural from urban nursing practice (Thornton 1992a, Thornton 1992b, Hegney 1996, Hegney et al. 1997). From these early studies onwards, the implications of living and working in the same community have been a key difference for rural nurses internationally (Bushy and Leipert 2005, Lauder et al. 2005, Richards et al. 2005).

Currently the issue of greatest concern for Australian rural nurses is recruitment and retention of staff (Crowley and West 2002). This trend that is reflected internationally (International Council of Nurses 2006). Strategies to improve the retention of nurses, including mentoring, have been implemented across a range of settings (McCloughen and O'Brien 2005, Mills et al. 2005), but the effectiveness of such initiatives has not been measured. Mentoring as a workforce support strategy has been adopted in health care and other environments in Australia because it is cost-effective, drawing on local resources. Many publications have focused on mentoring and nursing, with a particular emphasis on the processes used (Glass and Walter 2000, Morton-Cooper and Palmer 2000); however, there is a gap in the literature about rural nursing and mentoring, with only two 
articles located that discuss mentoring programmes for rural nurses (Hanson and Hilde 1989, Waters et al. 2003). What little there is to read about rural nurses and supportive relationships is a hybrid of mentoring, clinical supervision and preceptoring (Mills et al. 2005).

The problem of workforce shortages for Australian rural nurses prompted the introduction of mentoring into discourses about solutions to poor recruitment and retention rates (Mills et al. 2006a). The Australian Government has been instrumental in introducing the use of mentoring as a recruitment and retention strategy. Underpinned by research that demonstrates that rural students eventually return to their birthplace as working adults (Hegney et al. 2002, Hegney et al. 2002a), a scholarship programme was introduced in $\mathbf{2 0 0 0}$ for students from a rural or remote location who are enrolled in an undergraduate nursing course. In addition to financial support, each scholarship recipient is encouraged to join a formal mentoring program that matches them with an experienced rural or remote nurse with the aim of providing them with pastoral support and a role model. Experienced rural or remote nurses who volunteer to mentor an undergraduate nursing student are given the opportunity to attend a two-day mentor development workshop with the aim of increasing their confidence and level of skills (Mills et al. 2006b). Workshops are provided by the main professional organisation for Australian rural nurses Australian Rural Nurses and Midwives (ARNM) whose program evaluation demonstrated that participants find the experience very valuable on a variety of levels, identifying mentoring as a relationship that they can also develop in their workplaces as well by participating a the mentoring program for undergraduate nursing students (Lennon 2004).

\section{The study}

\section{Aim}

The aim of the study was to examine rural nurses' experiences of mentoring.

\section{Methodology}

A grounded theory approach was adopted, using theoretical sampling, concurrent data generation and analysis, constant comparative analysis techniques during coding, category saturation, reflective memoing, situational analysis mapping and frame analysis (Mills et al. 2006c, Mills et al. 2006d). The data were generated during 2004 and 2005

\section{Participants}

National advertising and the snowball technique were used to recruit participants. Data were. Nine Australian rural nurses were recruited from Tasmania (1), Victoria (2), New South Wales (4), Queensland (1) and Western Australia (1). All of the participants were women. Their years of rural nursing experience ranged from three to 33, with the average being 19 years. Eight of the participants had taken part in a mentor development programme and self-defined as rural nurses.

\section{Data collection}

Eleven interviews were conducted using a semi-structured aide-memoir that was adapted to reflect the ongoing analysis that occurred during the data generation phase of the study; two participants were interviewed twice. Each interview was digitally recorded and transcribed verbatim. Three of the interviews were carried out by telephone and the remainder were face-to-face. One participant 
engaged in ongoing email dialogue, teasing out and clarifying the meaning of codes originally constructed by the researcher (JM) immediately after the original face-to-face interview. Another participant critiqued situational maps constructed by the researcher as the basis for their second interview, literally tracing her own patterns of meaning on to the codes, categories and collective actors visually represented in this way at that time; this created another cycle of co-construction for the researcher, contributing to subsequent reconstruction.

\section{Establishing rigour}

Fundamental to situating our grounded theory study in a constructivist paradigm was ensuring the 'goodness' of the findings, incorporating situatedness, trustworthiness and authenticity (Emden and Sandelowski 1998, Tobin and Begley 2004). Using a reflexive research design, we incorporated a decision or audit trail throughout the life of the study (Koch 2006), situating the researcher as an author with an influential life history. The responsibility for the construction of a grounded theory from the co-constructions of data generation is ours. We trace how we create these constructions through the writing of reflective memos, which form the basis of our analysis (Hall and Callery 2001, Mills et al. 2006c).

We also agree with Charmaz (2006) that Glaser's criteria for judging grounded theory provides a framework for participants and readers to use when evaluating constructivist grounded theory studies. Glaser (1978) proposes four premises (1978, p. 4-5) to which we have adapted to a constructivist approach. Theorising must show a 'fit' between categories constructed and the data generated in the study and it needs to 'work' to contextualise, interpret and predict the data.

'Relevance' has to be demonstrated through the theory connecting to the actions co-constructed by participants and researchers. Finally, constructivist grounded theories need to be 'modifiable' to reflect the multiple, ever-changing truths and realities of actors in the social worlds being researched.

\section{Ethical Considerations}

The study was approved by a university ethics committee. Recruitment was carried out as described above and signed consent was obtained from all participants, who were free to withdraw from the study at any time. Pseudonyms have been used in reporting the findings.

\section{Data Analysis}

Consistent with grounded theory methods, data collection and analysis were concurrent. Theoretical sampling occurred on the basis of this ongoing analysis and interview aide memoirs were amended to reflect current constructions and questions that remained unanswered.

Each interview was transcribed and coded. Initially coding was line-by-line, which led to open coding and the construction of a series of categories, their properties and dimensions. Many of the codes were 'in vivo' codes (Glaser and Strauss 1967) or used participants' language that 'crystallise[d] and condense[d] meaning' (Charmaz 2006, p.57) transforming into category names such as 'live my work', anchoring our analysis in the participants' social world. 
At the same time, axial coding was performed to relate each category to the others, thus ensuring integration (Strauss and Corbin 1998). A story-line technique was used to construct the core category (Strauss and Corbin 1990).

In each analytical phase we used constant comparison of data to data and then data to categories until saturation was reached. After nine interviews no new categories could be constructed and data collected after this point fitted the existing schema.

Data were coded using the Text Analysis Markup System (@ 2004 Matthew Weinstein) qualitative data analysis software. Data were then imported into a Filemaker Developer $7^{\mathrm{TM}}$ database for further sorting. Reflective memoing and situational mapping techniques (Clarke 2005) were employed extensively during each analytical phase of the study. Mapping and diagramming was carried out using Inspiration 7 software ${ }^{\mathrm{TM}}$ (Inspiration Software Inc).

\section{Findings}

\section{Live My Work}

For rural nurses, 'live my work' entailed managing a complex web of relationships and interactions using multiple perspectives of self that included their roles as community members, nurses and healthcare consumers. Self in this study is defined as 'the organised set of internalised attachments, commitments, attributes, images and identifications with which a person creates a concept of self' (Charmaz 1991, p.72 emphasis added). The perspective of self that they conceptualised as nurses could not be isolated to interactions that occurred during the working day; therefore, rural nurses felt that they lived their work. This can be compared to, for example, nurses working in a large urban acute care hospital who interact with substantial numbers of people that they never see again, or never see outside of work. As Mary says, 'I guess here in a small community I say that we $-\mathrm{I}$, live my work. It's not something that you do between 9.00 and 5.00' (Aary: TM 8192).

Reasons why nurses were attracted to working in rural and remote areas focused on their life outside of work. Family, friends, having grown up in a region and wanting their children to enjoy the same sense of close community bring nurses 'home' from larger urban centres: 'I was going to go on the Flying Doctor but of course met a man, so we stayed in [country town] and had 3 children' (Wyn). Rural nurses were firstly members of the community and it is fair to suggest that rural nurses rarely leave the community when they finish their working lives (Hegney et al. 2002). Because of this, they want to see a continuation of high quality health service delivery. 
Rural nurses who were aware, through experience, of issues involved in managing their lives using multiple perspectives of self were more likely to cultivate and grow novice rural nurses. They did this using three lenses or frames of reference: culture, politics and clinical practice.

Cultivate and grow is the core category of the grounded theory resulting from this study. The conditions that this occurred under were, planned face-to-face, planned distant and accidental faceto-face. Properties of cultivating and growing ranged from preceptoring, accidental mentoring, mentoring to deep friendship, with each successive name reflecting higher levels of engagement and trust between the novice and a more experienced rural nurse. Property names adopted were also reflective of the time required to establish a higher degree of trust and engagement.

\section{RURAL NURSES' FRAMES OF REFERENCE}

\section{Culture}

Live my work was framed by rural nurses through lenses of culture, politics and clinical practice, each of which was dependent on their different perspectives of self. Explaining the culture - accepted norms and practices - of rural communities is integral to how rural nurses framed cultivating and growing new and novice rural nurses. Live my work meant all of the novice nurses' actions, both inside and outside work, affected how they were perceived as a nurse. Experienced rural nurses had a high degree of sensitivity to potential professional compromises that may occur in the course of daily living, where interactions often become everybody's knowledge. Wyn illustrated this:

I suppose in a rural community... everything that you do has more of an impact. Like, if you have a relationship or if you have a problem here everybody knows about it. It becomes more of a problem because it's so... well advertised... If you were an anonymous person in an anonymous setting you really wouldn't care for a start, but probably no one else would care either... It wouldn’t become everybody’s sort of knowledge. Not that I mean everybody knows about things, but a lot more people do...so you really do need...somebody who's in your corner if you have a crisis or something happens... If you have a fight or if you have an altercation with say a doctor, that doctor is probably somebody... that you go to church with... It actually is more of an impact... on your self esteem ... on your whole life really than if it was somebody that you didn't know... I think it's extremely important to have a mentor to keep everything in perspective...so you don't sort of fold under the emotional pressure of it all... You know the fact is that people are dying around you. People that you know, sometimes people that you know very well ... families you know. It is... more of a... demand on you in that way. negotiate from various perspectives in their interactions with others as they live their work. There 
are few strangers to rural nurses who live in small communities, meaning that the emotional impact of interactions in the course of nursing from "womb to tomb" (Hegney 1996) is often high as the relationships they have with people come from multiple perspectives of self. These can involve intimacy, friendship and a sharing of fundamental world-views, in turn leading to a higher emotional investment and greater reciprocity than urban nurses may share with their patients and colleagues. Mentoring was constructed as a way of supporting novice rural nurses through the experience of transgressing multiple perspectives in living the whole of their lives, as well as a way of flagging potential compromises before they occurred.

\section{Politics}

The problem of workforce for Australian rural nurses is one that is framed by rural nurses framed as a localised political fight for survival in the face of economic rationalisation and the centralisation of health care services (Francis 2005). Rural nurses again lived out multiple perspectives in this conflict. Firstly, they were members of the wider community and had concern with the overall welfare of the community. Secondly, they may have had skills in endangered areas such as orthopaedics and midwifery, meaning there was potential for them to become deskilled. Thirdly, they were possible consumers of the same endangered services.

Mentoring novice rural nurses enabled consciousness-raising about the cause of comprehensive localised service delivery in rural areas. Implicit in this consciousness-raising was teaching effective political actions that nurses can take. One such action was reframing the argument about service provision from an economic rationalist perspective to one of improved client or patient outcomes for those who are able to stay in the local communities. For instance, Alice says:

I feel that we're fighting for survival... you don’t do things enough to be skilled and... there seems to be a constant “you can’t do hip replacements in your facility because you only do 7 or 8 a year” ... they don't look at the fact that our patients are in their home town, that they actually do very well, that ... we don't have any infections, that they're home quickly. That's a constant battle for survival... with ... orthopaedic surgery even midwifery. You only have 50 deliveries a year therefore your midwife can't be skilled, even though we do up skilling courses... (Researcher) And how do you think mentoring is going to impact on that? (Alice) I think ... explaining the political things that we have to do as well as the nursing'.

Rural nurses also used mentoring to renegotiate the politics of difference between urban and rural nursing by consciousness-raising about the role of the rural nurse and their capabilities. Urban nurses' images of rural nursing and health care often led them to believe that clients were unable to access the same level of care in the country as they would in a major metropolitan centre.

'Sometimes we are very put down by our city colleagues. I mean we have had midwives ring from the tertiary centres and say "will you send somebody down so that we can show them how to 
gavage a bub", and I think, skills that we all have! And [urban nurses saying] "no we can't possibly send you back there"' (Alice).

Mentoring nurses who are planning on returning to urban environments, or are working in urban environments reframed the image of rural nursing practice from the urban myth that depicted it as low-level care to that of a specialist field of generalist advanced practice (Francis et al. 2002) because as Alice stated, 'we're not specialists in anything and we can have anything appear at the front door'.

\section{Clinical Practice}

Focusing on the culture of clinical environments experienced rural nurses used mentoring to protect novice rural nurses from other rural nurses guilty of perpetrating forms of horizontal violence (Roberts 1983) towards them. 'It's a hard profession in that sometimes we say nurses are cannibals, you know, they eat their young, and they can sometimes go into a small country town and really just be swallowed up... that support is just not there' (Bella).

Cultivate and growing are positive words that denoted the approach that rural nurse mentors take towards their mentees, they wanted to metaphorically "protect their young". Margaret framed her experiences of being mentored as a novice rural nurse using workplace culture in a casualty unit (i.e. emergency department) to contextualise and explained the relationship's initial impetus:

The old boss up there and he... sort of took me under his wing... All the girls... when I first went to Cas[ualty]... [I was] 28, they were all sort of late 30s married with children, so I actually had nothing in common with these women and... I had no experience in Cas[ualty] whatsoever and I'm there thrown in as the new girl. So...Cas[ualty] being Cas[ualty], such a volatile place... he sort of tucked me in under his wing without trying to alienate him and I from the rest of his colleagues and peers and friends... because they had worked together for... 20 years some of them.'

Demystifying local practice or ways of communication were important tasks that mentors undertook, providing explanations for others' behaviours as well as coaching to increase the novice's mastery of skills. Often allowances were made for community members and colleagues who behave in ways that could be understood by novice rural nurses as a personal criticism. Their actions and attitudes needed to be translated by experienced rural nurses in order to depersonalise threatening situations.

We've got a doctor who is superb...I've worked with him for a long time, I know that his stress levels can come across as a criticism of you and I just explain it to them, look he's wonderful, we love him ... I think that's important that they understand. (Alice) 
The touchstone of traditional hospital training is another barrier that experienced rural nurses worked to break down by demonstrating how they valued new and novice nurses' knowledge and skills, and the positive outcomes that their interactions had for themselves because 'to teach is to learn twice' (Kaye). Entrenched resistance to novice rural nurses who had gained their registration through tertiary preparation was an issue. Kaye tells of her own experience of this:

I’ve walked onto wards and... I've had some of them say "great, new face, new knowledge, you learn so much more at uni[versity] then we ever did” and they're accepting and they're willing to listen. but then I’ve had others that go “what would you know you've only been at uni[versity]”... And that can break... a younger person. (TM:2489)

Rural nurses are required to maintain an extensive range of skills in order to meet the changing needs of their communities, often with a much less sophisticated range of technological diagnostic support services than urban nurses have access to. Considering the 'very complex, generalist nature of the rural nurse's work' (Francis et al. 2002, p.170) maintaining competence was difficult and required a high level of commitment to meet ongoing educational needs (Blue 2002). Skill development under these conditions is therefore very dependent on experienced rural nurses such as Mary who 'because I love my work... I have taught by example and I guess that having a passion for developing staff and therefore teaching them by working with them... that's how my mentoring has been'. (TM: 1806)

\section{Discussion}

After reviewing the literature about mentoring, findings generated in this study have been found to support Stewart and Krueger's theoretical definition of mentoring in nursing:

Mentoring in nursing is a teaching - learning process acquired through personal experience within a one-to-one, reciprocal, career development relationship between two individuals diverse in age, personality, life cycle, professional status, and/or credentials. The nurse dyad relies on the relationship in large measure for a period of several years for professional outcomes, such as research and scholarship; an expanded knowledge and practice base; affirmative action; and/or career progression. Mentoring nurses tend to repeat the process with other nurses for the socialization of [clinicians] scholars and scientists into the professional community and for the proliferation of a body of nursing knowledge. (Stewart and Krueger 1996, p. 315)

This definition was the outcome of an evolutionary concept analysis that built on the original work of Yoder (1990). Live my work provides evidence to broaden the potential mentees (scholars and scientists) listed in this definition to include clinicians who also require socialisation into the professional community or social world that constitutes nursing practice. 


\section{Living and working in the same community}

Results from this study illustrate how rural nurses see themselves as members of their community, as well as being both consumers and providers of health care while, adding to the existing literature (Hegney 1996, Hegney et al. 2002, Bushy and Leipert 2005, LeSergent and Haney 2005) about living and working in the same community through identifying the multiple perspectives of self that rural nurses use to manage this issue. Shibutani, a symbolic interactionist, develops the notion of multiple perspective of self. He posits the idea that actors have a variety of perspectives they use as frames of reference depending on which societal group they find themselves with at the time. Each perspective organises a view of the world, which is taken for granted about the attributes of objects, of events and of human nature (Shibutani 1962).

Community embeddedness, a term coined by Lauder et al. (2001) describes the sharing of norms that necessitate rural nurses constructing multiple perspectives of self:

'[L]iving, shopping and socializing as members of the community in which they work... These factors, allied to shared social expectations and outlook between the majority of the community and the [rural] District Nurses, may be responsible for creating a sense of embeddedness' (Lauder et al. 2001, p. 339, emphasis added).

Live my work confirms these findings and adds another dimension through the construction of frames of reference used by rural nurses. We would argue that these frames - culture, politics and clinical practice - could be extrapolated from rural nursing to other rural healthcare professions such as medicine and allied health. In Australia, Aboriginal health workers are another important group of rural health care workers who also live their work. Rural healthcare professionals and workers share many common practice characteristics that are context-specific - one of which is a 'fish bowl existence, which prevents anonymity' (Blue 2002, p.200).

How rural nurses use their experience to help novice nurses manage the phenomenon of live my work is important for other rural health professionals to learn from. Particularly how they develop supportive relationships such as mentoring in order to cultivate and grow their colleagues through demystifying local culture and clinical mores while at the same time troubleshooting.

Rural nurses' multiple perspectives of self facilitate membership of a variety of reference groups within their communities. Usually these reference groups are complementary in their norms; however, conflict can arise for individuals when they have to choose between two competing perspectives. Shibutani $(1955,1962)$ contends that in making such choices individuals are influenced by those termed significant others. For novice rural nurses, their mentors take on the role of significant others through translation and leading by example. 


\section{Study Limitations}

This study was designed to be exploratory and descriptive and to generate a grounded theory about Australian rural nurses' experiences of mentoring. A limitation of the study originated from the initial design when we advertised for rural nurses to talk about their experiences of mentoring. Participants who volunteered had all attended mentor development workshops facilitated by one of us and this had led them to define some of the supportive relationships they developed in practice as mentoring relationships. Through snowball recruitment, we attracted one participant who had not had any formal mentoring training; however, the participant group were on the whole wellinformed about the possibilities of mentoring, which influenced both how they constructed their eligibility to participate and our co-constructions about their experiences.

\section{Conclusion}

Promoting understanding about what it means to live my work is an important challenge that arises from this study. Rurality is not confined to geographically large countries such as Australia, New Zealand, the USA and Canada, even highly urbanised European countries have places that are sparsely populated and have to meet the challenges of providing adequate access to health care services staffed by skilled clinicians. The recent establishment of the European Rural and Isolated Practitioners Association (EURIPA) is testament to this (Lionis and Worley 2003).

Our findings are important in informing local action to retain novice rural nurses in the workforce. Internationally, rural practice for a range of clinicians creates a far different set of demands both clinically and personally than urban practice. Recognising that mentoring encompasses and translates an extremely broad range of issues gives it a higher value than may currently be realised. 


\section{References}

Australian Institute of Health and Welfare (2004) Rural, regional and remote health: a guide to remoteness classifications. In Rural Health Series Number 4AIHW, Canberra.

Blue, I. (2002) Australian Rural Health Care Professional Practice. In The New Rural Health (Eds, Wilkinson, D. and Blue, I.) Oxford, Melbourne, pp. 190-203.

Bushy, A. and Leipert, B. (2005) Factors that influence students in choosing rural nursing practice: a pilot study. Rural and Remote Health, 5(387), Online.

Charmaz, K. (1991) Turning Points and Fictional Identities. In Social Organization and Social Process: Essays in honor of Anselm Strauss (Ed, Maines, D.) Aldine De Gruyter, New York, pp. 71-86.

Charmaz, K. (2006) Constructing Grounded Theory: A Practical Guide Through Qualitative Analysis, Sage, Thousand Oaks.

Clarke, A. (2005) Situational Analysis: Grounded Theory After the Postmodern Turn, Sage Publications, Thousand Oaks.

Crowley, R. and West, S. (2002) The Patient Profession: Time for Action - Report on the inquiry into nursing, Senate Community Affairs References Committee, Canberra, Australia.

Emden, C. and Sandelowski, M. (1998) The good, the bad and the relative, part one: Conceptions of goodness in qualitative research. International Journal of Nursing Practice, 4, $206-212$.

Francis, K. (2005) Health and Health Practice in Rural Australia: Where Are We, Where To From Here? Online Journal of Rural Nursing and Health Care, 5(1), http://www.rno.org/journal/issues/Vol-5/issue-1/Francis article.htm.

Francis, K., Bowman, D. and Redgrave, M. (2002) Knowledge and Skills Required by Rural Nurses to Meet the Challenges of a Changing Work Environment in the 21st Century: A review of the literature. In National Review of Nursing Education in 2002: Literature reviews Commonwealth of Australia, Canberra, pp. 154-215.

Glaser, B. (1978) Theoretical Sensitivity: Advances in the Methodology of Grounded Theory, Sociology Press, Mill Valley.

Glaser, B. and Strauss, A. (1967) The Discovery of Grounded Theory, Aldine De Gruyter, New York.

Glass, N. and Walter, R. (2000) An experience of peer mentoring with student nurses: enhancement of personal and professional growth. Journal of Nursing Education, 39(4), 155-60.

Hall, W. and Callery, P. (2001) Enhancing the Rigor of Grounded Theory: Incorporating Reflexivity and Relationality. Qualitative Health Research, 11(2), 257-272.

Hanson, C. M. and Hilde, E. (1989) Faculty mentorship: support for nurse practitioner students and staff within the rural community health setting. Journal of Community Health Nursing, 6(2), 73-81.

Hegney, D. (1996) The Status of Rural Nursing in Australia: A Review. Australian Journal of Rural Health, 4, 1-10.

Hegney, D. and McCarthy, A. (2002) Preparation for Rural Nursing Practice. In The New Rural Health (Eds, Wilkinson, D. and Blue, I.) Oxford University Press, Melbourne, pp. 237-252. 
Hegney, D., McCarthy, A., Rogers-Clark, C. and Gorman, D. (2002) Why nurses are attracted to rural and remote practice. Australian Journal of Rural Health., 10(3), 178-86.

Hegney, D., McCarthy, A., Rogers-Clark, C. and Gorman, D. (2002a) Retaining rural and remote area nurses: the Queensland, Australia experience. Journal of Nursing Administration., 32(3), 12835.

Hegney, D., Pearson, A. and McCarthy, A. (1997) The Role and Function of The Rural Nurse in Australia, Royal College of Nursing, Australia, Canberra.

Koch, T. (2006) Establishing rigour in qualitative research: the decision trail. Journal of Advanced Nursing, 53(1), 91-103.

Lauder, W., Reel, S., Farmer, J. and Griggs, H. (2005) Social capital, rural nursing and rural nursing theory. Nursing Inquiry, 13(1), 73-79.

Lauder, W., Reynolds, W., Reilly, V. and Angus, N. (2001) The role of district nurses in caring for people with mental health problems who live in rural settings. Journal of Clinical Nursing, 10, 337-344.

International Council of Nurses (2006) The Global Nursing Shortage: Priority Areas for Intervention. Geneva, pp. 63.

Lennon, D. (2004) Mentor Development and Support Project: Achievements and Outcomes March 2003-2004. Association for Australian Rural Nurses Canberra.

LeSergent, C. and Haney, C. (2005) Rural hospital nurse's stressors and coping strategies: a survey. International Journal of Nursing Studies, 42, 315-324.

Lionis, C. and Worley, P. (2003) Emergence of the European Section of the international journal of Rural and Remote Health. Rural and Remote Health, 3 (online), 257.

McCloughen, A. and O'Brien, L. (2005) Development of a mentorship programme for new graduate nurses in mental health. International Journal of Mental Health Nursing, 14, 276-284.

Mills, J., Francis, K. and Bonner, A. (2006a) The problem of workforce for the social world of Australian rural nurses: a collective action frame analysis. Journal of Nursing Management, Accepted for publication September 2006.

Mills, J., Lennon, D. and Francis, K. (2006b) Mentoring Matters: developing rural nurses knowledge and skills. Collegian, 13(32-36).

Mills, J., Bonner, A. and Francis, K. (2006c) Adopting a Constructivist Approach to Grounded Theory: Implications for Research Design. International Journal of Nursing Practice, 12(1), 8-13.

Mills, J., Bonner, A. and Francis, K. (2006d) The Development of Constructivist Grounded Theory. International Journal of Qualitative Methods, 5(1), Article 3.

Mills, J., Francis, K. and Bonner, A. (2005) Mentoring, clinical supervision and preceptoring: clarifying the conceptual definitions for Australian Rural Nurses. A review of the literature. Rural and Remote Health, 5(410), Online. 
Morton-Cooper, A. and Palmer, A. (2000) Mentoring, preceptorship and clinical supervision, Blackwell Science Ltd., Oxford.

Richards, H., Farmer, J. and Selvaraj, S. (2005) Sustaining the rural primary healthcare workforce: survey of healthcare professionals in the Scottish Highlands. Rural and Remote Health, 5(365), Online.

Roberts, S. (1983) Oppressed group behavior: implications for nursing. Advances in Nursing Science, 5(4), 21-30.

Shibutani, T. (1955) Reference Groups as Perspectives. The American Journal of Sociology, 60(6), 562-569.

Shibutani, T. (1962) Reference Groups and Social Control. In Human Behavior and Social Processes: An Interactionist Approach (Ed, Rose, A.) Routledge \& Kegan Paul, London.

Stewart, B. M. and Krueger, L. E. (1996) An evolutionary concept analysis of mentoring in nursing. Journal of Professional Nursing, 12(5), 311-21.

Strauss, A. and Corbin, J. (1990) Basics of Qualitative Research: Grounded Theory Procedures and Techniques, Sage Publications, Newbury Park.

Strauss, A. and Corbin, J. (1998) Basics of Qualitative Research: Techniques and Procedures for Developing Grounded Theory, Sage Publications, Thousand Oaks.

Thornton, R. (1992a) Rural Nursing Practice. In Issues in Rural Nursing (Ed, Courtney, M.) University of New England, Armidale, pp. 65-69.

Thornton, R. (1992b) Rural Nursing Practice. In Issues in Australian Nursing 3(Eds, Gray, G. and Pratt, R.) Churchill Livingstone, Melbourne, pp. 121-132.

Tobin, G. and Begley, C. (2004) Methodological rigour within a qualitative framework. Journal of Advanced Nursing, 48(4), 388-396.

Vance, C. and Olson, R. (1998) The Mentor Connection in Nursing, Springer Publishing, New York.

Waters, D., Clarke, M., Harris Ingall, A. and Dean-Jones, M. (2003) Evaluation of a pilot mentor programme for nurse managers. Journal of Advanced Nursing, 42(5), 516-526.

Yoder, L. (1990) Mentoring: a concept analysis. Nursing Administration Quarterly, 15(1), 9-19. 